\title{
The mTOR Signaling Pathway Is Associated With the Prognosis of Malignant Pleural Mesothelioma After Multimodality Therapy
}

\author{
AYUMI KURODA ${ }^{1}$, SEIJI MATSUMOTO ${ }^{1}$, AKIHIRO FUKUDA $^{1,2}$, TORU NAKAMICHI ${ }^{1}$, \\ AKIFUMI NAKAMURA ${ }^{1}$, MASAKI HASHIMOTO ${ }^{1}$, TERUHISA TAKUWA ${ }^{1,2}$, \\ NOBUYUKI KONDO ${ }^{1}$, TOHRU TSUJIMURA ${ }^{3}$, TAKASHI NAKANO ${ }^{4}$ and SEIKI HASEGAWA ${ }^{1}$ \\ ${ }^{1}$ Department of Thoracic Surgery, Hyogo Collage of Medicine, Nishinomiya, Japan; \\ ${ }^{2}$ Department of Thoracic Surgery, Saiseikai Noe Hospital, Osaka, Japan; \\ ${ }^{3}$ Department of Pathology, Hyogo Collage of Medicine, Nishinomiya, Japan; \\ ${ }^{4}$ Center for Respiratory Disease, Otemae Hospital, Osaka, Japan
}

\begin{abstract}
Background/Aim: We performed multimodality therapy comprising preoperative chemotherapy, extrapleural pneumonectomy (EPP), and radiation therapy for patients with malignant pleural mesothelioma (MPM). Although multimodality therapy resulted in good prognosis, further improvement is required. Therefore, herein, we analysed the prognostic factors using surgical specimens and searched for suitable molecular targets to improve the prognosis after multidisciplinary treatment. Patients and Methods: Forty-six patients with MPM underwent multimodality therapy. Paraffinembedded surgical samples were used for immunohistochemistry to evaluate the expression of phosphorylated (p-) AKT, extracellular signal-regulated kinase (ERK), mammalian target of rapamycin (mTOR), mitogen-activated protein kinase (MAPK), eukaryotic translation initiation factor $4 E$-binding protein 1 (4E-BP1), and S6 ribosomal protein $(S 6 R P)$. Results: On univariate and multivariate analyses, significant differences were observed according to the histological type, pathological stage, and p-mTOR expression rate. Conclusion: The prognosis of MPM is affected by p-mTOR expression, suggesting that molecular-targeted treatment might be used during multimodal therapy for MPM.
\end{abstract}

Malignant pleural mesothelioma (MPM) is a rare disease (13 ), and the median survival of untreated patients is $4-12$

Correspondence to: Seiji Matsumoto, Department of Thoracic Surgery, Hyogo College of Medicine, 1-1 Mukogawa-cho, Nishinomiya, Hyogo 663-8501, Japan. Tel: +81 798456885, Fax: +81 798456897, e-mail: smatumo@hyo-med.ac.jp

Key Words: mTOR signal pathway, malignant pleural mesothelioma, extrapleural pneumonectomy. months (4-6). The occurrence of MPM is strongly associated with asbestos exposure, and MPM develops after a latent period of 20-40 years (2-7). First-line therapy consists of combination therapy using cisplatin and pemetrexed, which results in better outcomes compared to the outcomes after cisplatin monotherapy $(8,9)$. However, cisplatin and pemetrexed combination therapy only extends the median survival by $9-12$ months (8). Therefore, multimodal therapy comprising preoperative chemotherapy, extrapleural pneumonectomy (EPP), and radiation therapy is currently performed $(10,11)$. Although multimodal therapy results in good results, with an overall median survival of 19.9 months and a 2-year survival rate of $42.9 \%$, EPP leaves minute tumors on the surface of the resected stump due to the irregular nature of chest wall detachment (12). Therefore, postoperative chemotherapy using cisplatin and pemetrexed or radiotherapy is required. In addition, as there are only a few effective anticancer drugs for MPM, new drugs are needed to improve the prognosis of patients with MPM.

The mammalian target of rapamycin (mTOR) and mitogenactivated protein kinase (MAPK) pathways play important roles in responding to extracellular signals from cytokines and growth factors, as well as regulating cell function $(13,14)$; these pathways are also known to be overactivated in some sarcomas (15). While BRAF inhibitors and mitogen-activated protein kinase kinase (MEK) inhibitors targeting the MAPK pathway, and mTOR inhibitors targeting the phosphatidylinositol-4,5-bisphosphate 3-kinase (PI3K)/AKT serine/ threonine kinase (AKT)/mTOR pathway are currently in use, only a few studies have evaluated the extent of overactivation of these pathways in MPM cases.

Therefore, the current study investigated the possibility of using molecular-targeted drugs as multimodal therapy for MPM by analyzing the correlation between AKT/mTOR and 
MAPK pathways and prognosis of MPM cases treated with multimodal therapy including EPP.

\section{Patients and Methods}

Patients and treatment. Multimodal therapy including EPP was administered to 46 patients with MPM at our Department between April 2004 and October 2012. After the diagnosis of MPM on pleural biopsy, three courses of chemotherapy $\left(75 \mathrm{mg} / \mathrm{m}^{2}\right.$ cisplatin and $500 \mathrm{mg} / \mathrm{m}^{2}$ pemetrexed every 21 days) were administered and EPP was performed for patients who did not show any disease progression. The same chemotherapy was continued after surgery. The tumors were staged using the International Mesothelioma Interest Group (IMIG) staging system (16). This study was approved by The Ethics Review Board for Human Genome/Gene Analysis Research, Hyogo College of Medicine (no. 0044), and informed consent was obtained from each patient prior to evaluating the specimens.

Immunohistochemistry. Immunohistochemistry was performed using intra-operatively obtained paraffin-embedded tissue samples. Formalin-fixed specimens were embedded in paraffin and cut into 3$\mu \mathrm{m}$ slices. They were then immersed in a buffer solution of $\mathrm{pH} 9$ (DAKO, Carpinteria, CA, USA) and kept at $98^{\circ} \mathrm{C}$ for 20 minutes before being cooled for 20 minutes to stimulate the antigens. The following rabbit monoclonal antibodies were used as primary antibodies: phosphorylated ( $p$-) AKT (Ser473; 1:50), p-mammalian target of rapamycin (mTOR) (Ser2448; 1:100), p-S6 ribosomal protein (S6RP) (Ser240/244; 1:2000, 1:1600), p-eukaryotic translation initiation factor 4E-binding protein 1 (4E-BP1) (Thr37/46; 1:400), pMEK (Ser221; 1:50), and p-ERK (Thr202/Tyr204; 1:400) (Cell Signaling Technology, Danvers, MA, USA). A DAKO Autostainer (Ft. Collins, CO, USA) was used for staining. The expression of $\mathrm{p}-$ AKT, p-mTOR, p-S6RP, p-4E-BP1, p-MEK and p-ERK were analyzed by the intensity of staining by two researchers who were blinded to the patients' baseline characteristics.

Statistical analysis. Continuous and categorical variables are presented as the median values with ranges and as frequencies with percentages, respectively. The curves for overall survival from the time of surgery were estimated using the Kaplan-Meier productlimit method, and were compared using the log-rank test for univariate analysis. Factors that were found to have values of $p<0.1$ on univariate analysis were included in the Cox proportionalhazards regression model for multivariate analysis. The results are presented as the hazards ratios (HRs), 95\% confidence intervals (CI), and $p$-values. All $p$-values were two-sided, and values of $p<0.05$ were considered to indicate statistical significance. Statistical analyses were performed using SPSS Statistics software (version 15.0; SPSS Inc., Chicago, IL, USA).

\section{Results}

Multimodal therapy including EPP was administered to 46 patients with MPM: 35 men (76.1\%) and 11 women (23.9\%), with a mean age of 59.8 years (range=37-71 years). Histological type was epithelial in the majority of cases $(93.5 \%)$. The disease stage was stage III or IV in most cases (71.7\%) (Table I).
Table I. Clinical characteristics of patients with malignant pleural mesothelioma $(N=46)$.

\begin{tabular}{lc}
\hline Characteristic & Value \\
\hline Gender, n (\%) & \\
Male & $35(76.1 \%)$ \\
Female & $11(23.9 \%)$ \\
Age, years & $59.8(37-71)$ \\
Median & \\
Histology, n (\%) & $43(93.5 \%)$ \\
Epithelial type & $2(4.3 \%)$ \\
Biphasic type & $1(2.2 \%)$ \\
Sarcomatoid type & \\
Side, n $(\%)$ & $27(58.7 \%)$ \\
Left & $19(41.3 \%)$ \\
Right & \\
IMIG pStage, n (\%) & $2(4.3 \%)$ \\
1 & $11(23.9 \%)$ \\
2 & $28(60.9 \%)$ \\
3 & $5(10.9 \%)$ \\
4
\end{tabular}

IMIG: International Mesothelioma Interest Group; p-Stage: pathological stage.

The results of immunohistochemical staining are shown in Table II. While the rate of positive expression for $\mathrm{p}-\mathrm{AKT}, \mathrm{p}$ mTOR, and p-ERK was approximately $40 \%$, the rates for $\mathrm{p}$ S6RP and p-MEK were relatively high, at $69.6 \%$ and $87.0 \%$, respectively (Table II).

No correlation was observed between the prognosis of patients and the positivity for $\mathrm{p}$-AKT, $\mathrm{p}$-4EBP1, and p-ERK ( $p=0.925, p=0.650$, and $p=0.647$, respectively). Although of borderline significance, the prognosis of the p-mTORpositive group was better than that of the p-mTOR-negative group (median 37.1 vs. 14.4 months, $p=0.085$ ). In contrast, the prognosis of the p-MEK-negative group was better than that of the p-MEK-positive group (not reached $v s .17 .4$ months, $p=0.084$ ). Moreover, the prognosis of the p-S6RPpositive group was significantly better than that of the $\mathrm{p}$ S6RP-negative group (43.6 vs. 14.4 months, $p=0.031$; Figure 1).

On univariate analysis, epithelioid histological type (vs. non-epithelioid) led to significantly poorer prognosis $(\mathrm{HR}=4.328,95 \% \mathrm{CI}=1.366-13.715 ; p=0.013)$. The prognosis was also significantly worse for those with p-stage IV $(\mathrm{HR}=3.698,95 \% \mathrm{CI}=1.231-11.115 ; p=0.02)$ and but better for those with positive $\mathrm{p}-\mathrm{S} 6 \mathrm{RP}$ expression $(\mathrm{HR}=0.435,95 \%$ $\mathrm{CI}=0.2-0.946 ; p=0.036)$.

On multivariate analysis, only two out of the three abovementioned factors remained significant: epithelioid histological type $(\mathrm{HR}=3.617,95 \% \quad \mathrm{CI}=1.039-12.592$; $p=0.043)$, and $\mathrm{p}$-Stage IV $(\mathrm{HR}=5.782,95 \% \mathrm{CI}=1.57-21.299$; $p=0.008$ ) (Table III). 
Table II. Results of immunohistochemical staining $(N=46)$.

\begin{tabular}{lcrr}
\hline Antigen & Protein name & Positive cases, n (\%) & Negative cases, n (\%) \\
\hline AKT & Protein kinase B & $18(39.1 \%)$ & $28(60.9 \%)$ \\
mTOR & Mammalian target of rapamycin & $18(39.1 \%)$ & $28(60.9 \%)$ \\
S6RP & S6 ribosomal protein & $32(69.6 \%)$ & $14(30.4 \%)$ \\
4E-BP1 & Eukaryotic translation initiation factor 4E-binding protein 1 & $27(58.7 \%)$ & $19(41.3 \%)$ \\
MEK & Mitogen-activated protein kinase kinase & $40(87.0 \%)$ & $6(13.0 \%)$ \\
ERK & Extracellular signal-regulated kinase & $19(41.3 \%)$ & $27(58.7 \%)$ \\
\hline
\end{tabular}

\section{Discussion}

The results of the current study showed that the prognosis of patients with MPM was influenced by p-mTOR expression, suggesting that molecular-targeted therapy has the potential to be used as part of multimodal therapy for MPM.

MAPK signaling cascades are linked to RAS, RAF, MEK, and ERK and play an important role in the proliferation of cells and resistance to anticancer drugs (17). Therefore, methods that block the MAPK pathway are being evaluated as a possible method for cancer treatment. The U.S. Food and Drug Administration has approved combination treatment with BRAF and MEK inhibitors such as dabrafenib and trametinib, vemurafenib and cobimetinib, and encorafenib and binimetinib for $B R A F^{\mathrm{V} 600}$-mutated advanced melanoma. The combination of dabrafenib and trametinib can be used after surgery for stage III melanoma. Vemurafenib was the first molecular-targeted drug to show a survival benefit for $B R A F^{\mathrm{V} 600 \mathrm{E}}$ metastatic melanoma. Vemurafenib was superior to dacarbazine when used as first-line treatment for $B R A F^{\mathrm{V} 600 \mathrm{E}}$-mutant metastatic melanoma. The objective response rate (ORR) after vemurafenib treatment was $48 \%$ versus $5 \%$ after dacarbazine treatment, while the median progression-free survival (PFS) rate was 5.3 versus 1.6 months, respectively (18).

Selective MEK inhibitors can inhibit the growth of $B R A F$ and NRAS-mutant melanoma. Trametinib was the first MEK inhibitor approved for the treatment of $B R A F$-mutated metastatic melanoma not previously treated with BRAF inhibitors. The ORR after trametinib treatment was $22 \%$ versus $8 \%$ after dacarbazine treatment, and the median PFS was 4.8 months versus 1.5 months for BRAF-mutant metastatic melanoma, respectively (19). Thus, MEK inhibitor therapy in combination with a BRAF inhibitor is more effective and less toxic than treatment with a BRAF inhibitor alone. In addition, when BRAF inhibitor or MEK inhibitor monotherapy is used, negative feedback through ERK1 and ERK2 can be an issue (20).

Approximately $50 \%$ of melanomas have $B R A F$ mutations, with the V600E missense mutation accounting for approximately $80-90 \%$. In 1,046 samples from patients who underwent radical surgery for primary non-small cell lung carcinoma, $B R A F$ mutations were detected in 37 tumors (3.5\%) and the BRAF mutation of V600E was observed in $56.7 \%$ of cases (21). Approximately $4 \%$ of MPM cases have $B R A F$ mutations, and most of these mutations result in the substitution of BRAF V600E (22).

In the current study, the rate of positive expression of $\mathrm{p}$ MEK was relatively high $(87.0 \%)$, and the prognosis of the p-MEK-negative group was better than that of the p-MEKpositive group (not reached versus 17.4 months, $p=0.084$ ). In fact, for MPM, when the frequency of the $B R A F \mathrm{~V} 600 \mathrm{E}$ gene mutation is low (22), we expect MEK inhibitors to be effective because there are many p-MEK-positive cases.

In patients with mesothelioma, the level of hyaluronic acid (HA) in serum and pleural fluid is high, probably because HA is produced by both mesothelioma cells and normal mesothelial cells. HA, via receptors, modulates the intracellular signaling pathways, thereby resulting in cell proliferation, increased motility, and higher invasive properties of malignant cells (23). The inhibitor of HA synthesis 4-methylumbelliferone suppresses the growth of mesothelioma cell lines, and is effective for MPM treatment when combined with trametinib (24).

In the current study, the histological type, and p-Stage were prognostic factors after multidisciplinary treatment for MPM. In addition, p-mTOR expression was a prognostic factor on univariate analysis, and we propose a method that uses an mTOR inhibitor in combination with cisplatin/pemetrexed as a possible improvement. The first mTOR inhibitor to be discovered was rapamycin. At the time of discovery, rapamycin also showed immunosuppressive activity, and was therefore used as an immunosuppressant during organ transplantation. In addition, rapalogs including temsirolimus, everolimus, and ridaforolimus are currently used as anticancer agents, owing to the cytostatic effect of rapamycin on mTORC1 inhibition (25).

In a previous study, the $\mathrm{p}$-mTOR positive rate was high in early-stage epithelial type mesothelioma and in the biphasic, sarcomatoid type, while the p-mTOR positive rate decreased in late stage MPM; in addition, overall survival tended to be 
A

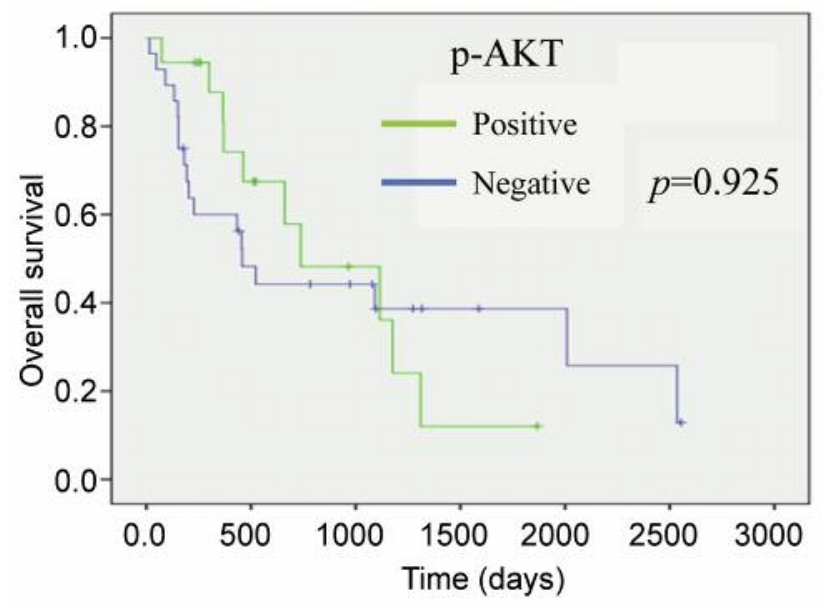

C

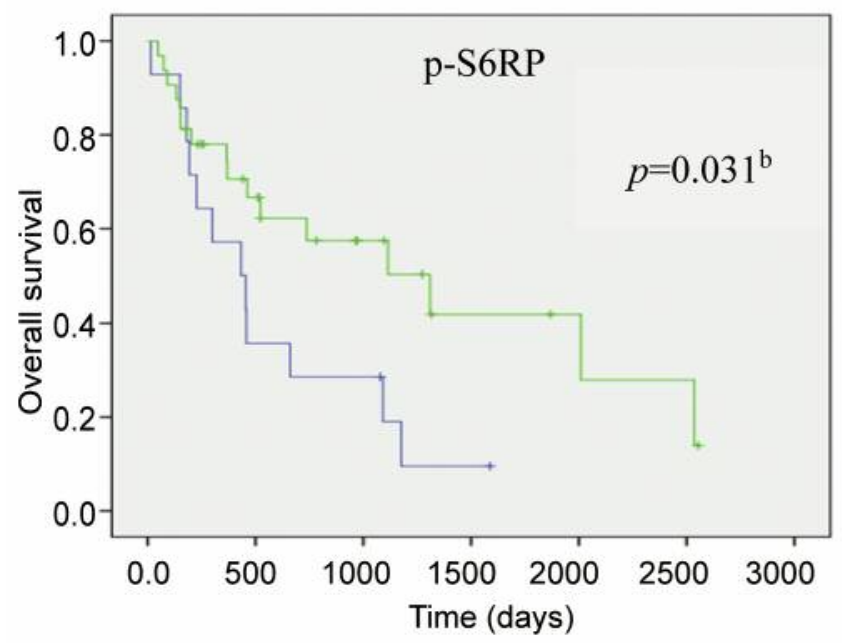

E

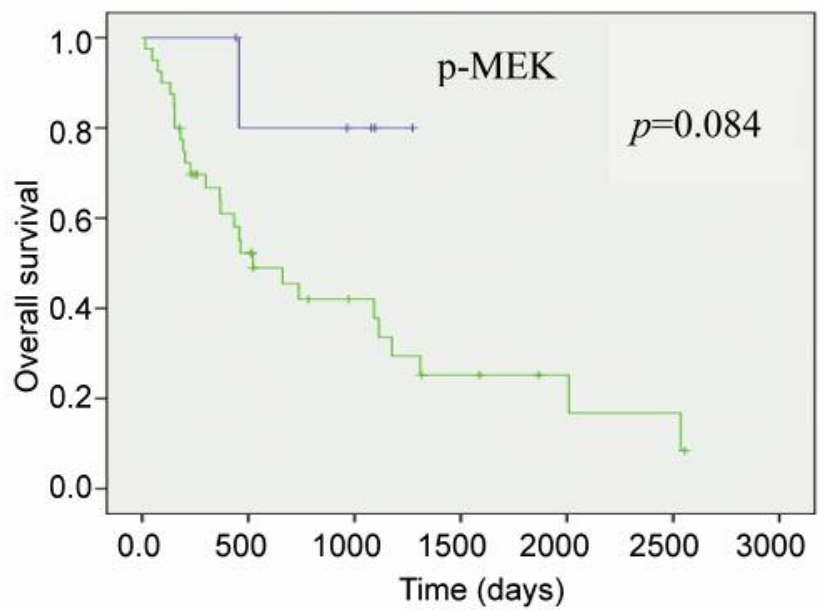

B

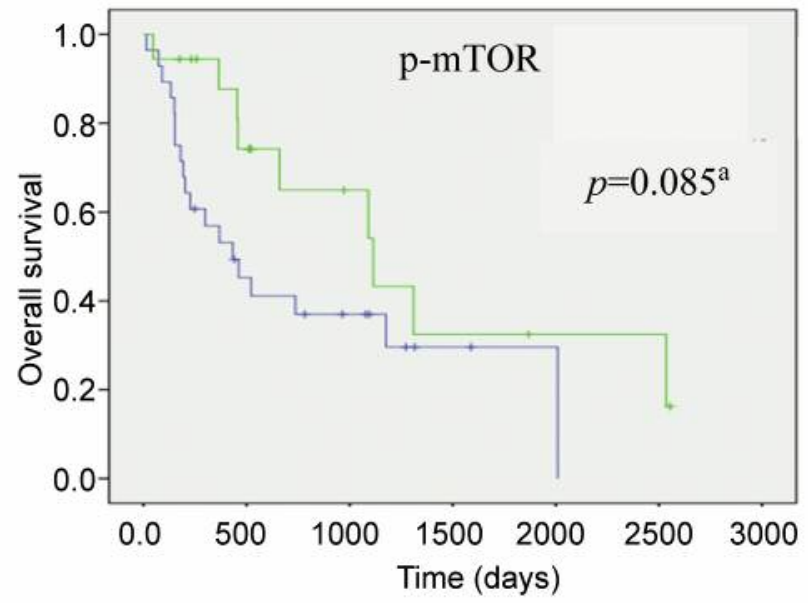

D

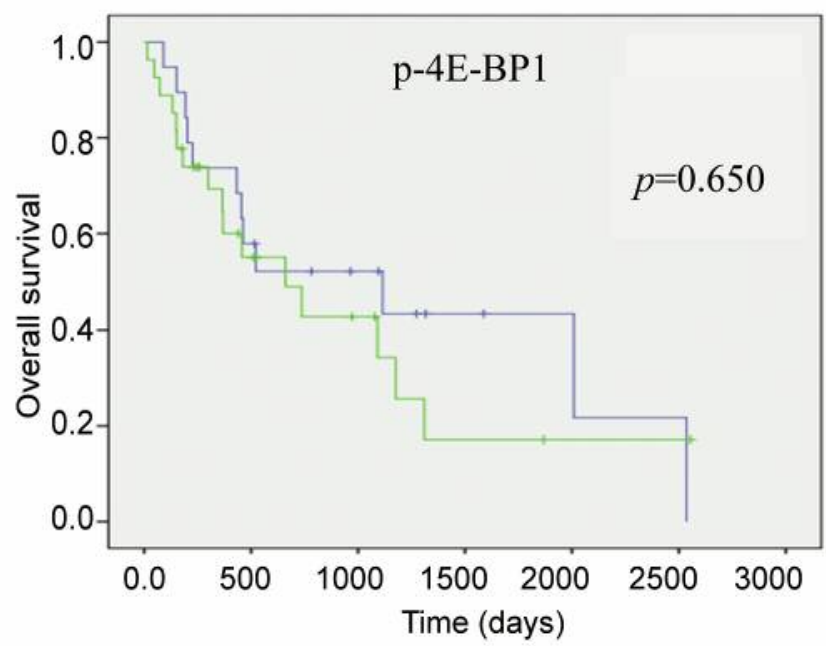

F

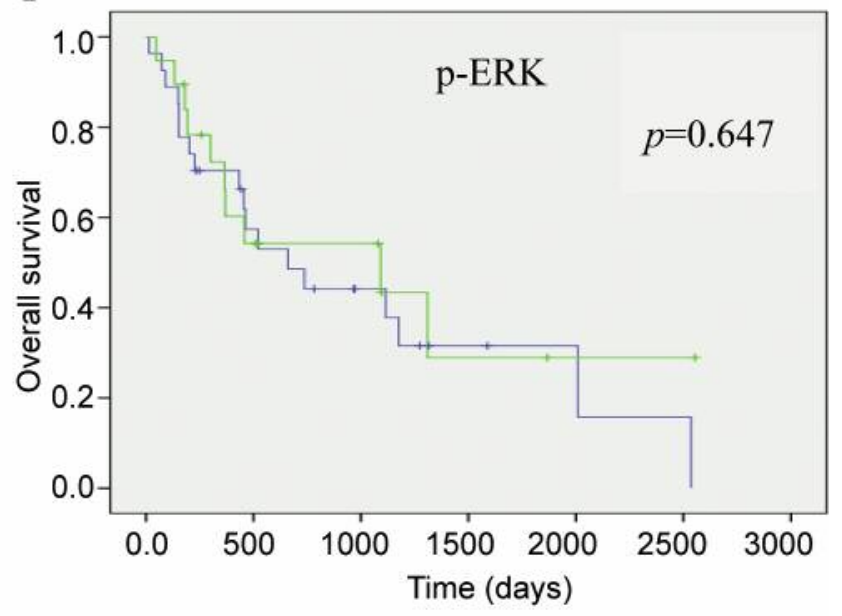

Figure 1. Kaplan-Meier analysis of overall survival according to positivity for phosphorylated ( $p$-) forms of protein kinase ( $p$-AKT) (A), mammalian target of rapamycin ( $p$-mTOR) (B), S6 ribosomal protein ( $p$-S6RP) (C), eukaryotic translation initiation factor 4E-binding protein 1 ( $p$-4E-BP1) $(D)$, mitogen-activated protein kinase kinase $(p-M E K)(E)$, and extracellular signal-regulated kinase ( $p$-ERK) $(F)$. p-Values when adjusted for multivariable factors: ${ }^{a} p=0.107$ and ${ }^{b} p=0.314$. 
Table III. Univariate and multivariate analyses for preoperative factors in patients with malignant pleural mesothelioma $(N=46)$.

\begin{tabular}{|c|c|c|c|c|}
\hline \multirow[b]{2}{*}{ Factor } & \multicolumn{2}{|c|}{ Univariate analysis } & \multicolumn{2}{|c|}{ Multivariate analysis } \\
\hline & HR 95\% CI & $p$-Value & HR 95\% CI & $p$-Value \\
\hline \multicolumn{5}{|l|}{ Age } \\
\hline$>60$ Years & 1 & & & \\
\hline$\leq 60$ Years & $0.416(0.182-0.949)$ & 0.037 & $0.716(0.313-1.637)$ & 0.428 \\
\hline \multicolumn{5}{|l|}{ Gender } \\
\hline Male & 1 & & & \\
\hline Female & $0.453(0.155-1.322)$ & 0.147 & & \\
\hline \multicolumn{5}{|l|}{ Side } \\
\hline Left & 1 & & & \\
\hline Right & $0.847(0.387-1.852)$ & 0.677 & & \\
\hline \multicolumn{5}{|l|}{ Histology } \\
\hline Epithelioid & 1 & & 1 & \\
\hline Non-epithelioid & $4.328(1.366-13.715)$ & 0.013 & $3.617(1.039-12.592)$ & 0.043 \\
\hline \multicolumn{5}{|l|}{ IMIG p-Stage } \\
\hline I, II, III & 1 & & 1 & \\
\hline IV & $3.698(1.231-11.115)$ & 0.02 & $5.782(1.57-21.299)$ & 0.008 \\
\hline \multicolumn{5}{|l|}{ p-S6RP } \\
\hline Negative & 1 & & 1 & \\
\hline Positive & $0.435(0.200-0.946)$ & 0.036 & $0.640(0.268-1.525)$ & 0.314 \\
\hline \multicolumn{5}{|l|}{ p-mTOR } \\
\hline Negative & 1 & & 1 & \\
\hline Positive & $0.489(0.213-1.123)$ & 0.091 & $0.471(0.189-1.175)$ & 0.107 \\
\hline \multicolumn{5}{|l|}{ p-MEK } \\
\hline Negative & 1 & & & \\
\hline Positive & $4.921(0.664-36.464)$ & 0.119 & & \\
\hline \multicolumn{5}{|l|}{ p-4EBP1 } \\
\hline Negative & 1 & & & \\
\hline Positive & $0.832(0.375-1.845)$ & 0.650 & & \\
\hline \multicolumn{5}{|l|}{ p-AKT } \\
\hline Negative & 1 & & & \\
\hline Positive & $0.954(0.355-2.564)$ & 0.925 & & \\
\hline \multicolumn{5}{|l|}{ p-ERK } \\
\hline Negative & 1 & & & \\
\hline Positive & $0.834(0.384-1.812)$ & 0.647 & & \\
\hline
\end{tabular}

CI: Confidence intervaI; HR: hazard ratio; IMIG: International Mesothelioma Interest Group; p-Stage: pathological stage; p-S6RP: phosphorylated S6 ribosomal protein; p-MEK: phosphorylated mitogen-activated protein kinase kinase; p-mTOR: phosphorylated mammalian target of rapamycin; p-4EBP1: phosphorylated eukaryotic translation initiation factor 4E-binding protein 1; p-AKT: phosphorylated protein kinase B; p-ERK: phosphorylated extracellular signal-regulated kinase.

better in the p-mTOR-positive group than in the p-mTORnegative group (26). In the current study, multimodal treatment with neoadjuvant chemotherapy (cisplatin and pemetrexed) was successful for treating early-stage MPM, consistent with the results of the previous study.

Based on the results of this study, we believe that mTOR inhibitors would contribute to the improvement of prognosis in the treatment of mesothelioma. However, mTOR inhibitors alone are ineffective, and it is desirable to use an mTOR inhibitor in combination with cisplatin/pemetrexed. In the current study, univariate and multivariate analyses revealed that $\mathrm{p}$-mTOR expression was a prognostic factor, and the results suggest that the use of mTOR inhibitors is promising. Based on the same hypothesis, clinical trials of mTOR inhibitors were conducted for MPM. The SWOG S0722 trial evaluated the efficacy and safety of everolimus for patients with mesothelioma after chemotherapy with platinum-based regimens. The ORR was $2 \%$; the median PFS was 2.8 months, and the median overall survival was 6.3 months (27). Because mTOR inhibitors alone are less effective against mesothelioma than other cancer types, combination therapy with chemotherapy is used. Sirolimus and cisplatin alone or in combination inhibited the growth of mesothelioma cell lines (28). Temsirolimus and cisplatin in combination were effective for mesothelioma, and temsirolimus induced apoptosis in cisplatin-resistant 
mesothelioma cell lines (26). In a previous study, we showed that everolimus or selumetinib alone had significant antitumor activity, and the combination of everolimus and selumetinib enhanced the individual antitumor activity in MPM xenograft models (29). We believe that the combination of mTOR inhibitors with other drugs is desirable rather than using mTOR inhibitors alone.

During multimodal therapy for MPM, preoperative chemotherapy is initially administered and surgery is only performed for patients who respond to the treatment. In the present study, the significant differences observed p-mTOR expression suggest that molecular-targeted therapy can be used during multimodal therapy for MPM.

\section{Conflicts of Interest}

The Authors have no conflicts of interest to declare regarding this study.

\section{Authors' Contributions}

SM and SH designed the study. AK wrote the main article text and prepared the Figure. AF, TN, AN, MH, TT and NK were involved with sample collection. SM performed the data analysis. TT, TN provided project supervision. All Authors approved the final article.

\section{Acknowledgements}

The Authors would like to thank Editage (www.editage.com) for English language editing.

\section{References}

1 Robinson BW, Musk AW and Lake RA: Malignant mesothelioma. Lancet 366(9483): 397-408, 2005. PMID: 16054941. DOI: 10.1016/S0140-6736(05)67025-0

2 Robinson BW and Lake RA: Advances in malignant mesothelioma. N Engl J Med 353(15): 1591-1603, 2005. PMID: 16221782. DOI: 10.1056/NEJMra050152

3 Stayner L, Welch LS and Lemen R: The worldwide pandemic of asbestos-related diseases. Annu Rev Public Health 34: 205-216, 2013. PMID: 23297667. DOI: 10.1146/annurev-publhealth031811-124704

4 Alberts AS, Falkson G, Goedhals L, Vorobiof DA and Van der Merwe CA: Malignant pleural mesothelioma: A disease unaffected by current therapeutic maneuvers. J Clin Oncol 6(3): 527-535, 1988. PMID: 3351560. DOI: 10.1200/JCO.1988. 6.3 .527

5 Branscheid D, Krysa S, Bauer E, Bulzebruck H and Schirren J: Diagnostic and therapeutic strategy in malignant pleural mesothelioma. Eur J Cardiothorac Surg 5(9): 466-472; discussion 473, 1991. PMID: 1931091. DOI: 10.1016/10107940(91)90142-7

6 Schildge J, Kaiser D, Henss H, Fiebig H and Ortlieb H: Prognostic factors in diffuse malignant mesothelioma of the pleura. Pneumologie 43(11): 660-664, 1989. PMID: 2608642.
7 Peto J, Decarli A, La Vecchia C, Levi F and Negri E: The European mesothelioma epidemic. Br J Cancer 79(3-4): 666672, 1999. PMID: 10027347. DOI: 10.1038/sj.bjc.6690105

8 Vogelzang NJ, Rusthoven JJ, Symanowski J, Denham C, Kaukel E, Ruffie P, Gatzemeier U, Boyer M, Emri S, Manegold C, Niyikiza $\mathrm{C}$ and Paoletti P: Phase III study of pemetrexed in combination with cisplatin versus cisplatin alone in patients with malignant pleural mesothelioma. J Clin Oncol 21(14): 26362644, 2003. PMID: 12860938. DOI: 10.1200/JCO.2003.11.136

9 van Meerbeeck JP, Gaafar R, Manegold C, Van Klaveren RJ, Van Marck EA, Vincent M, Legrand C, Bottomley A, Debruyne C, Giaccone G, European Organisation for Research and Treatment of Cancer Lung Cancer Group, and National Cancer Institute of Canada: Randomized phase III study of cisplatin with or without raltitrexed in patients with malignant pleural mesothelioma: An intergroup study of the European Organisation for Research and Treatment of Cancer Lung Cancer Group and the National Cancer Institute of Canada. J Clin Oncol 23(28): 6881-6889, 2005. PMID: 16192580. DOI: $10.1200 / J C O .20005 .14 .589$

10 Jackman DM: Current options for systemic therapy in mesothelioma. Semin Thorac Cardiovasc Surg 21(2): 154-158, 2009. PMID: 19822287. DOI: 10.1053/j.semtcvs.2009.06.010

11 Marshall JL and Andrews PA: Preclinical and clinical experience with cisplatin resistance. Hematol Oncol Clin North Am 9(2): 415-429, 1995. PMID: 7642471.

12 Hasegawa S, Okada M, Tanaka F, Yamanaka T, Soejima T, Kamikonya N, Tsujimura T, Fukuoka K, Yokoi K, and Nakano T: Trimodality strategy for treating malignant pleural mesothelioma: Results of a feasibility study of induction pemetrexed plus cisplatin followed by extrapleural pneumonectomy and postoperative hemithoracic radiation (Japan Mesothelioma Interest Group 0601 Trial). Int J Clin Oncol 21(3): 523-530, 2016. PMID: 26577445. DOI: 10.1007/s10147015-0925-1

13 Altomare DA and Testa JR: Perturbations of the AKT signaling pathway in human cancer. Oncogene 24(50): 7455-7464, 2005. PMID: 16288292. DOI: 10.1038/sj.onc.1209085

14 Vakiani E and Solit DB: KRAS and BRAF: Drug targets and predictive biomarkers. J Pathol 223(2): 219-229, 2011. PMID: 21125676. DOI: $10.1002 /$ path.2796

15 Endo M, Yamamoto H, Setsu N, Kohashi K, Takahashi Y, Ishii T, Iida K, Matsumoto Y, Hakozaki M, Aoki M, Iwasaki H, Dobashi Y, Nishiyama K, Iwamoto Y and Oda Y: Prognostic significance of AKT/mTOR and MAPK pathways and antitumor effect of mTOR inhibitor in NF1-related and sporadic malignant peripheral nerve sheath tumors. Clin Cancer Res 19(2): 450-461, 2013. PMID: 23209032. DOI: 10.1158/1078-0432.CCR-12-1067

16 Rusch VW: A proposed new international TNM staging system for malignant pleural mesothelioma. From the International Mesothelioma Interest Group. Chest 108(4): 1122-1128, 1995. DOI: 10.1378/chest.108.4.1122

17 Burotto M, Chiou VL, Lee JM, and Kohn EC: The MAPK pathway across different malignancies: A new perspective. Cancer 120(22): 3446-3456, 2014. PMID: 24948110. DOI: 10.1002/cncr.28864

18 Chapman PB, Hauschild A, Robert C, Haanen JB, Ascierto P, Larkin J, Dummer R, Garbe C, Testori A, Maio M, Hogg D, Lorigan P, Lebbe C, Jouary T, Schadendorf D, Ribas A, O'Day SJ, Sosman JA, Kirkwood JM, Eggermont AM, Dreno B, Nolop K, Li J, Nelson B, Hou J, Lee RJ, Flaherty KT, McArthur GA 
and BRIM-3 Study Group: Improved survival with vemurafenib in melanoma with $B R A F$ V600E mutation. N Engl J Med 364(26): 2507-2516, 2011. PMID: 21639808. DOI: 10.1056/ NEJMoa1103782

19 Flaherty KT, Robert C, Hersey P, Nathan P, Garbe C, Milhem M, Demidov LV, Hassel JC, Rutkowski P, Mohr P, Dummer R, Trefzer U, Larkin JM, Utikal J, Dreno B, Nyakas M, Middleton MR, Becker JC, Casey M, Sherman LJ, Wu FS, Ouellet D, Martin AM, Patel K, Schadendorf D and METRIC Study Group: Improved survival with MEK inhibition in $B R A F$-mutated melanoma. N Engl J Med 367(2): 107-114, 2012. PMID: 22663011. DOI: 10.1056/NEJMoa1203421

20 Johnson GL, Stuhlmiller TJ, Angus SP, Zawistowski JS and Graves LM: Molecular pathways: Adaptive kinome reprogramming in response to targeted inhibition of the BRAFMEK-ERK pathway in cancer. Clin Cancer Res 20(10): 25162522, 2014. PMID: 24664307. DOI: $10.1158 / 1078-0432 . C C R-$ 13-1081

21 Marchetti A, Felicioni L, Malatesta S, Grazia Sciarrotta M, Guetti L, Chella A, Viola P, Pullara C, Mucilli F and Buttitta F: Clinical features and outcome of patients with non-small-cell lung cancer harboring BRAF mutations. J Clin Oncol 29(26): 3574-3579, 2011. PMID: 21825258. DOI: 10.1200/JCO 2011.35.9638

22 Mezzapelle R, Miglio U, Rena O, Paganotti A, Allegrini S, Antona J, Molinari F, Frattini M, Monga G, Alabiso O and Boldorini R: Mutation analysis of the EGFR gene and downstream signalling pathway in histologic samples of malignant pleural mesothelioma. Br J Cancer 108(8): 1743-1749, 2013. PMID: 23558893. DOI: 10.1038/bjc.2013.130

23 Toole BP: Hyaluronan-CD44 interactions in cancer: Paradoxes and possibilities. Clin Can Res 15(24): 7462-7468, 2009. PMID: 20008845. DOI: 10.1158/1078-0432.CCR-09-0479

24 Cho H, Matsumoto S, Fujita Y, Kuroda A, Menju T, Sonobe M, Kondo N, Torii I, Nakano T, Lara PN, Gandara DR, Date H and Hasegawa S: Trametinib plus 4-methylumbelliferone exhibits antitumor effects by ERK blockade and CD44 downregulation and affects PD-1 and PD-L1 in malignant pleural mesothelioma. J Thorac Oncol 12(3): 477-490, 2017. PMID: 27867002. DOI: $10.1016 /$ j.jtho. 2016.10 .023
25 Zhou H and Huang S: Role of mTOR signaling in tumor cell motility, invasion and metastasis. Curr Protein Pept Sci 12(1): 30-42, 2011. PMID: 21190521 PMCID: PMC3410744

26 Hoda MA, Mohamed A, Ghanim B, Filipits M, Hegedus B, Tamura M, Berta J, Kubista B, Dome B, Grusch M, Setinek U, Micksche M, Klepetko W and Berger W: Temsirolimus inhibits malignant pleural mesothelioma growth in vitro and in vivo: Synergism with chemotherapy. J Thorac Oncol 6(5): 852-863, 2011. PMID: 21358348. DOI: 10.1097/JTO.0b013e31820e1a25

$27 \mathrm{Ou}$ SH, Moon J, Garland LL, Mack PC, Testa JR, Tsao AS, Wozniak AJ and Gandara DR: SWOG S0722: phase II study of mTOR inhibitor everolimus (RAD001) in advanced malignant pleural mesothelioma (MPM). J Thorac Oncol 10(2): 387-391, 2015. PMID: 25611229. DOI: 10.1097/JTO.0000000000000360

28 Hartman ML, Esposito JM, Yeap BY and Sugarbaker DJ: Combined treatment with cisplatin and sirolimus to enhance cell death in human mesothelioma. J Thorac Cardiovasc Surg 139(5): 1233-1240, 2010. PMID: 19853261. DOI: 10.1016/j.jtcvs.2009. 06.027

29 Matsumoto S, Orui H, Kuroda A, Hashimoto M, Yoneda K, Takuwa T, Kondo N and Hasegawa S: Combination efficacy of mTOR and MEK inhibitor in malignant pleural mesothelioma (MPM). J Clin Oncol 31: e18557-e18557, 2017. DOI: 10.1200 /jco.2013.31.15_suppl.e18557
Received August 30, 2019

Revised September 20, 2019

Accepted September 25, 2019 